\title{
INTRODUCCIÓN AL PROYECTO BIOMIMÉTICO
}

Caterina Mele

Department of Structural, Geotechnical and Building Engineering (DISEG). Universidad Politécnica de Turín. Italia.

E-mail: caterina.mele@polito.it

La Biomimética es la ciencia que, a partir de competencias multi e interdisciplinarias, estudia el funcionamiento de los organismos naturales para extraer principios para aplicar en formas, procesos, sistemas y estrategias útiles para resolver problemas humanos en modo sostenible.

La aplicación de principios biomiméticos en el proyecto de arquitectura puede ser un camino interesante, aún por explorar, con el objetivo de realizar edificios innovativos con bajo impacto ambiental.

Tres días intensos con relatores internacionales con diversas competencias interdisciplinares, visitas guiadas, ejemplos y trabajos grupales para explorar un enfoque rico de ideas aun inexploradas y con un gran potencial.

Que quiere decir Biomimética (Bios Mimesis = vida + imitación). Es la Ciencia que, "estudia los sistemas biológicos naturales emulando formas, procesos, mecanismos de acción, estrategias, para resolver los desafíos de cada día, para encontrar soluciones sostenibles a los problemas proyectuales y tecnológicos del hombre, para replicar dibujos y procesos en nuevas soluciones tecnológicas para la industria y la investigación" así la define Janine Benyus, la bióloga norteamericana que ha fundado el Biomimicry Institute. En 1958 el ingeniero aeronáutico Jack Steele había acuñado la palabra "Biónica" definiendo "una ciencia de los sistemas que funcionan basándose en sistemas naturales", pero la Biomimética non se limita a imitar la forma o la estructura de los organismos vivientes, sino que extrae los principios de funcionamiento que son fundamentales en la vida y para el éxito evolutivo de los sistemas biológicos.

El termino Biomimesis fue utilizado por la primera vez por el físico Otto Schmitt en el 1968 para indicar "una disciplina que simula las estructuras biológicas para realizar productos eficientes", pero solamente a partir de los años '80 y '90 comienza a enseñarse en la universidad y en centros de investigación de diferentes países entre los cuales el Centre of biomimetics de la Universidad de Reading y de Universidad de Bath en Inglaterra, en Friburgo donde nacen los cursos de Biomimesis, en los USA, donde nacen numerosos centros de investigación, formación y consultoría (el CBID-Centre for biologically inspired design, el Biomimicry Institute 3.8 en Montana fundado por Benyus). Actualmente es una ciencia en expansión también en China han sido fundados centros de investigación sobre Biomimética.

"Cada cosa que puedes imaginar, la naturaleza la ha ya creado": lo sostenía Albert Einstein, durante milenios los artesanos $e$ inventores de todo tipo se han inspirado 
libremente a partir de este enfoque, proponiendo formas y soluciones inspiradas en la Naturaleza.

Pero el cambio de paradigma es pasar desde: Learning about the natural world hacia Learning from the natural world, that's the switch. That's the profound switch." Janine Benyus ha profundizado y divulgado eficazmente estos temas.

Desde siempre el hombre se ha inspirado en la Naturaleza para encontrar soluciones eficaces y eficientes para sobrevivir en el planeta y para aumentar el proprio confort y la calidad de vida. En la proyectación de las manufacturas el hombre ha continuamente imitado estructuras, formas, proporciones geométricas, colores, ritmos, simetrías, funciones de los organismos biológicos con diferentes finalidades.

Aún ahora el desarrollo de los conocimientos científicos siempre más profundos, junto al desarrollo de instrumentos tecnológicos como las nanociencias y las nanotecnologías permiten estudiar los fenómenos y materiales en escala atómica y molecular donde las propiedades difieren notablemente de las observadas en escala mayor, pero tales conocimientos se prestan aún para ser utilizados en escala diferente para aplicaciones, procesos y productos caracterizados por nuevas prestaciones en un número ilimitado de sectores.

Por lo tanto hoy podemos imitar la Naturaleza no solo en aspectos morfológicos sino en sus modelos estratégicos, organizativos y de proceso, extremadamente eficientes y realmente sostenibles (Ej. autoreparaciones, auto montaje, resiliencia etc.).

También el debate sobre la sostenibilidad ambiental hace emerger la conciencia por un lado la necesidad de cambiar en modo radical nuestro modelo de desarrollo en modo de abatir en modo drástico el consumo de recursos naturales y energético, del otro lado es claro que los cambios en la dirección de la sostenibilidad son aun lentos e ineficientes. Se tiene la conciencia que debiéramos reducir el consumo de los recursos naturales un $90 \%$ de los actuales, pero en realidad cada ano estamos consumiendo siempre más lo que la Naturaleza logra regenerar. (Overshoot day 2017 fue el 2 agosto, y cada año la fecha está siempre más cerca).

¿Cómo hacemos las cosas?

Ciclo de vida di un producto, muestra que cada fase de extracción, producción, transporte, distribución, consumo hay un grande gasto de energía, liberación de contaminantes y residuos (ejemplos de plástico en el mar y de los desechos informáticos en Guiyu en China). 

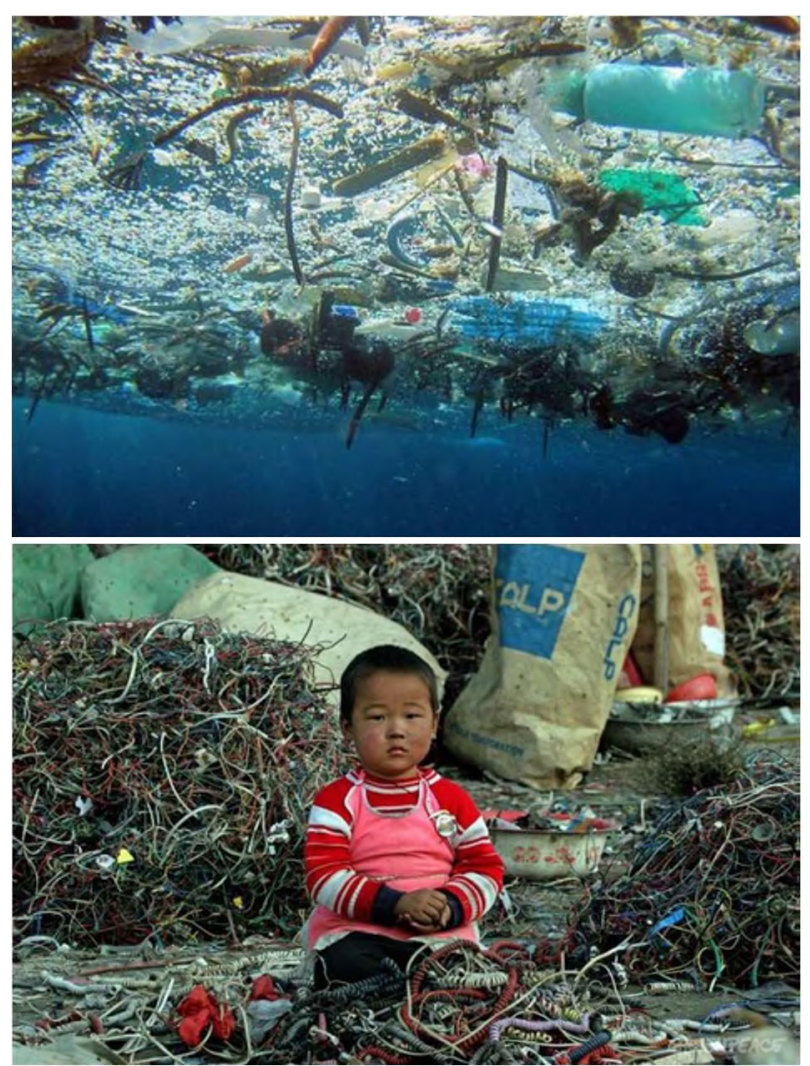

Fig. 1 Plástico en el mar y desechos electrónicos en Guiyu en China

\section{¿Cómo lo hace la Naturaleza?}

La Naturaleza no malgasta y no contamina y la vida cuida la Vida, crea las condiciones para favorecer la continuidad misma de la Vida. Los organismos vivos no producen desechos y utilizan una cantidad mínima de energía (mínima inversión para el máximo rendimiento) posible para sus actividades con el fin de garantizar las mejores prestaciones para conservar la especie.

En el enfoque biomimético la naturaleza es vista como Modelo (Model), Medida (Measure), y como Guía (Mentor) de la proyectación de los objetos y de las manufacturas técnicas.

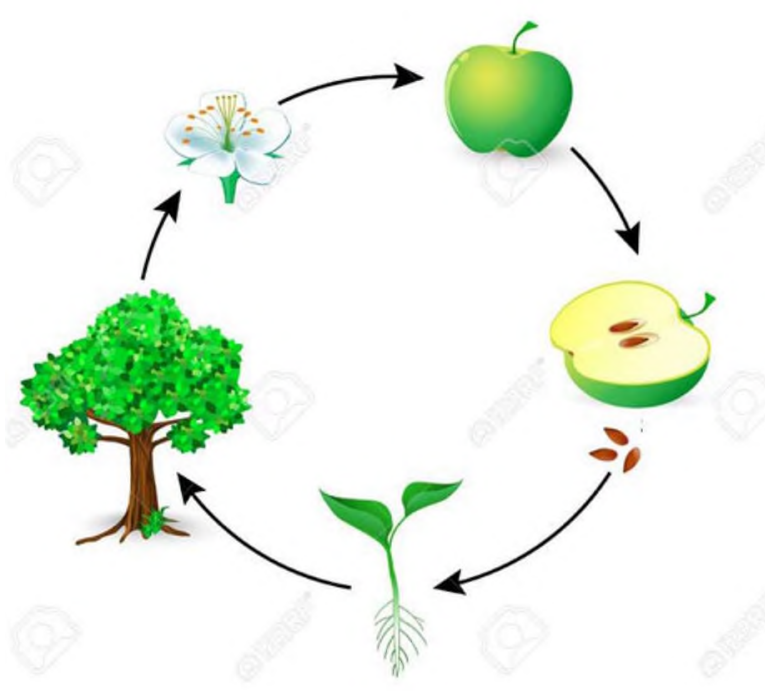

Fig. 2 La energía que se utiliza es solo renovable 
Todos los sistemas naturales respetan algunos principios fundamentales:

- Funcionan según ciclos cerrados: no existe el concepto de desecho

- se fundan en inventiva, interconexión, cooperación, procesos que fundan todos los sistemas

- funcionan con energía solar

- respetan y multiplican la diversidad.

El gran desafío de una sociedad humana verdaderamente sostenible podría encontrar soluciones que se han experimentado solamente adaptándose a la naturaleza, que es un banco de datos antigua con más de 4 millones de años de soluciones proyectuales innovativas, como dice Janine Benyus "un laboratorio de investigación y desarrollo potente y a nuestra disposición".
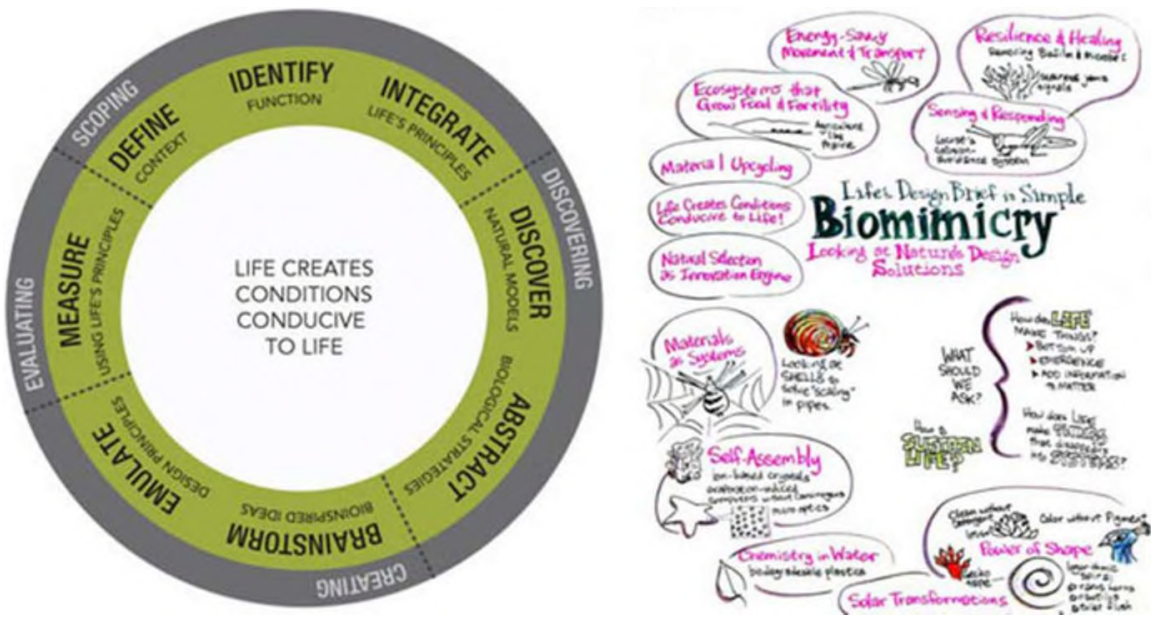

Fig. 3 Esquema del Instituto de biomimética, Janine Benyus

Por esto el enfoque biomimético parece muy prometedor.

Sería además necesario crear y aumentar los bancos de datos accesibles de innovación bioinspiradas en modo de transferir las soluciones en diferentes ámbitos de la tecnología humana, y aprender a afrontar un problema proyectual preguntándose cómo ha resuelto ese problema la naturaleza, con cual expediente y con cual proceso (¿cómo hace la Naturaleza?, ¿Cómo hacemos nosotros?) Esto implica la creación de grupos interdisciplinarios para la proyectación integrando conocimientos biológicos y técnicos.

Desde siempre el hombre trata de imitar a la naturaleza:

Uno de los primeros en inspirarse a la biomimética fue Leonardo Da Vinci, cuándo en sus estudios sobre la máquina volante tomaba como ejemplo el vuelo de los pájaros.

Pero también en la arquitectura en algún modo se ha siempre tomado como referencia la naturaleza: el capitel y la columna (árbol, cesto de hojas de acanto) el techo del Crystal Palace de Londres, construido a mitad del siglo XIX del arquitecto y botánico Joseph Paxton e inspirado en la Victoria Amazónica, una planta perteneciente a la familia de las ninfas. La arquitectura orgánica y anatómica de Antoni Gaudi (Sagrada Familia, Casa Batlò, Parc Guell, ecc.) 


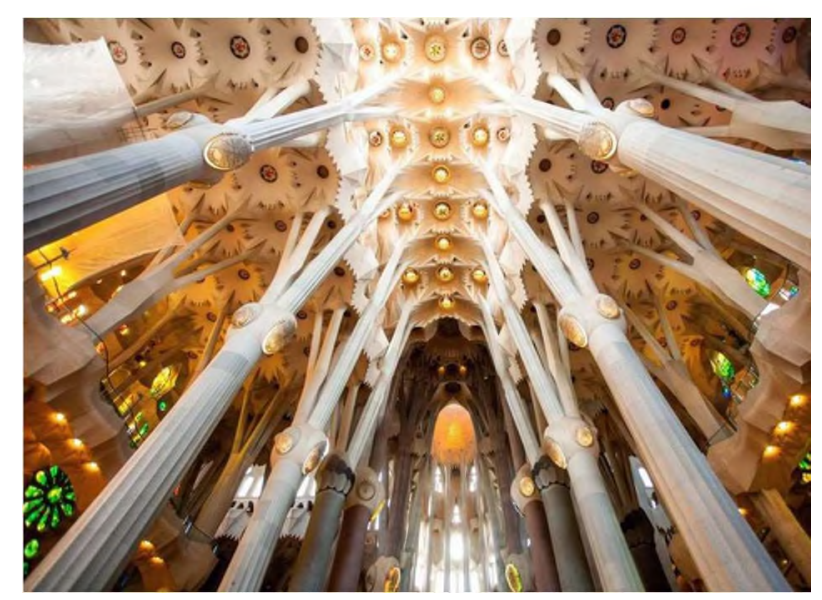

Fig. 4 sagrada Familia. Antonio Gaudì, Barcelona (iniciada en 1882)

Y en Paris el mismo Eiffel, por la necesidad de alivianar, lo más posible, la estructura de la Torre ha tomado idea de estudios del cuerpo humano, en particular de la estructura y del funcionamiento del fémur.
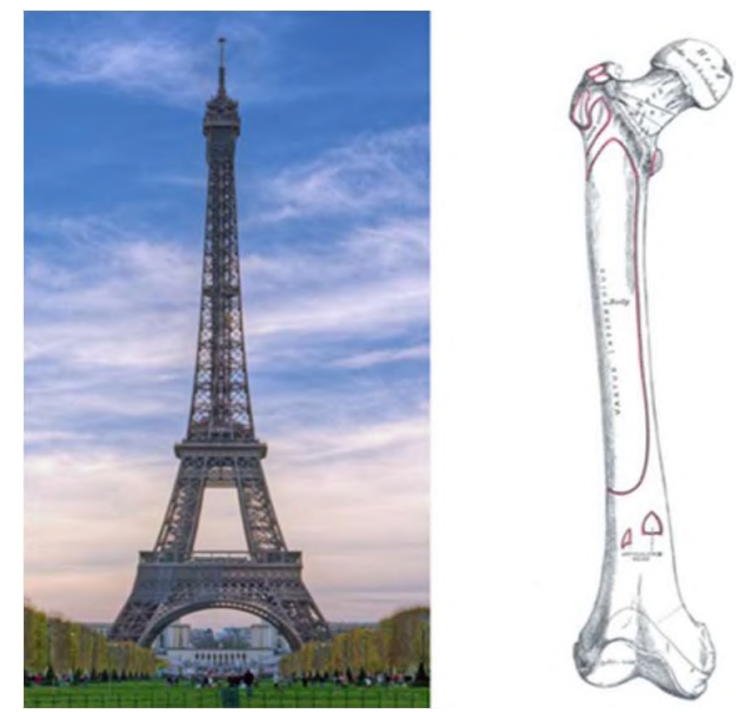

Fig. 5 Tour Eiffel. Su estructura nace a partir de estudios de la anatomía del fémur humano

Y aún más cerca nuestro, la arquitectura del Ingeniero y arquitecto Santiago Calatrava que se inspira casi siempre en la anatomía de seres vivientes (Estacion TGV a Lyone).

Aun en estos casos la imitación de la Naturaleza se detiene esencialmente en formas y estructuras. 


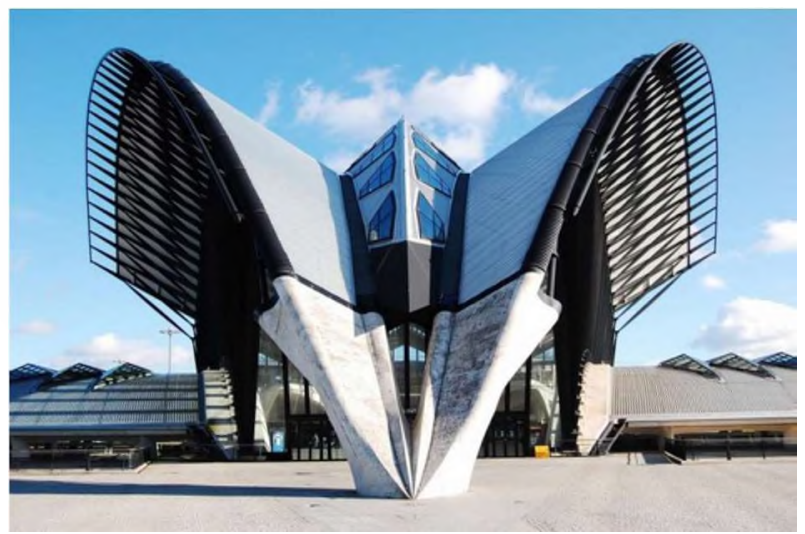

Fig. 6 Santiago Calatrava. Estación del TGV, Lyon, 1999

El enfoque biomimético actual implica la transferencia de soluciones técnicas adoptadas por organismos naturales para resolver un problema (ahorro hídrico, esparcir el calor, adhesión, construcción de un refugio, etc.

Un gran número de aplicaciones inspiradas en la naturaleza se ha traducido en aplicaciones técnicas y son comercialmente disponibles, como el velcro, las superficies autolimpiantes inspiradas en las hojas de loto (Nelumbo nucifera) y los adhesivos estructurales, desarrollados a partir del geco o de moluscos moribundos. ".Cada cosa que puedes imaginarte, la naturaleza la ha ya inventado".

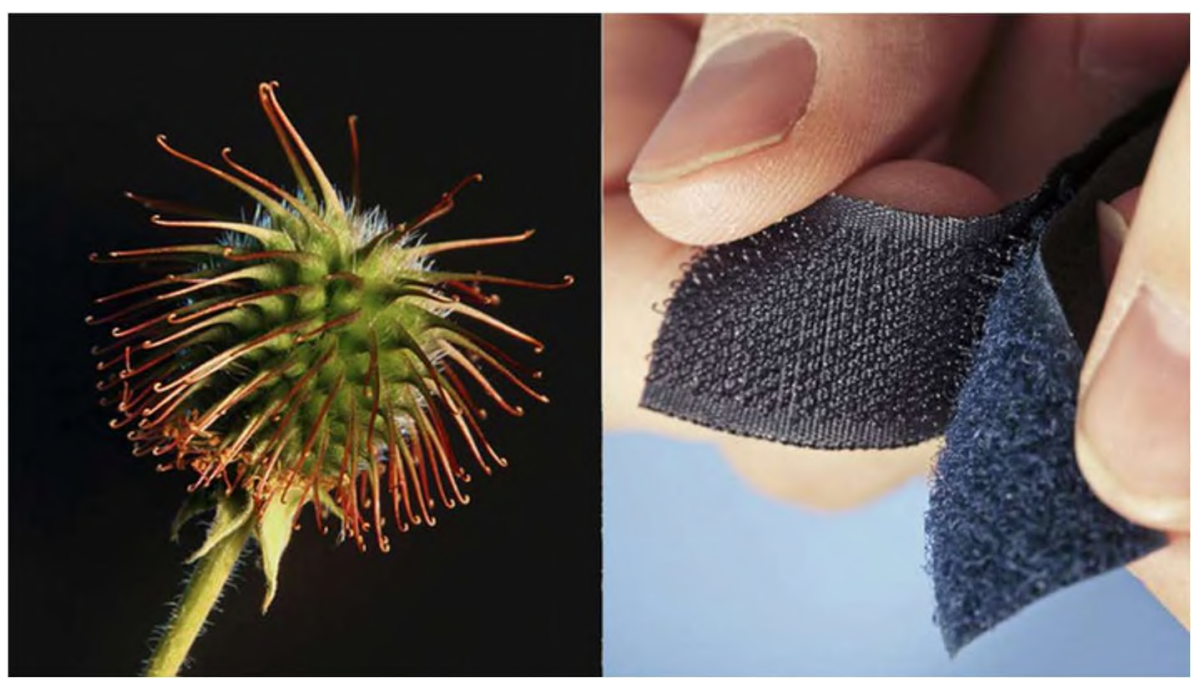

Fig. 6 Inspiración del velcro

La cala y su estructura a forma de espiral, permite almacenar y distribuir el agua según la necesidad de la planta. Hay filtros que imitan la estructura de la cala.

La arquitectura biomimética promete potencialidades completamente nuevas:

Como la Fachada Viviente de Chao Chen que reacciona con la lluvia, sin necesidad de ayudas mecánicas.

Chao Chen en su proyecto se ha inspirado completamente en la naturaleza. 

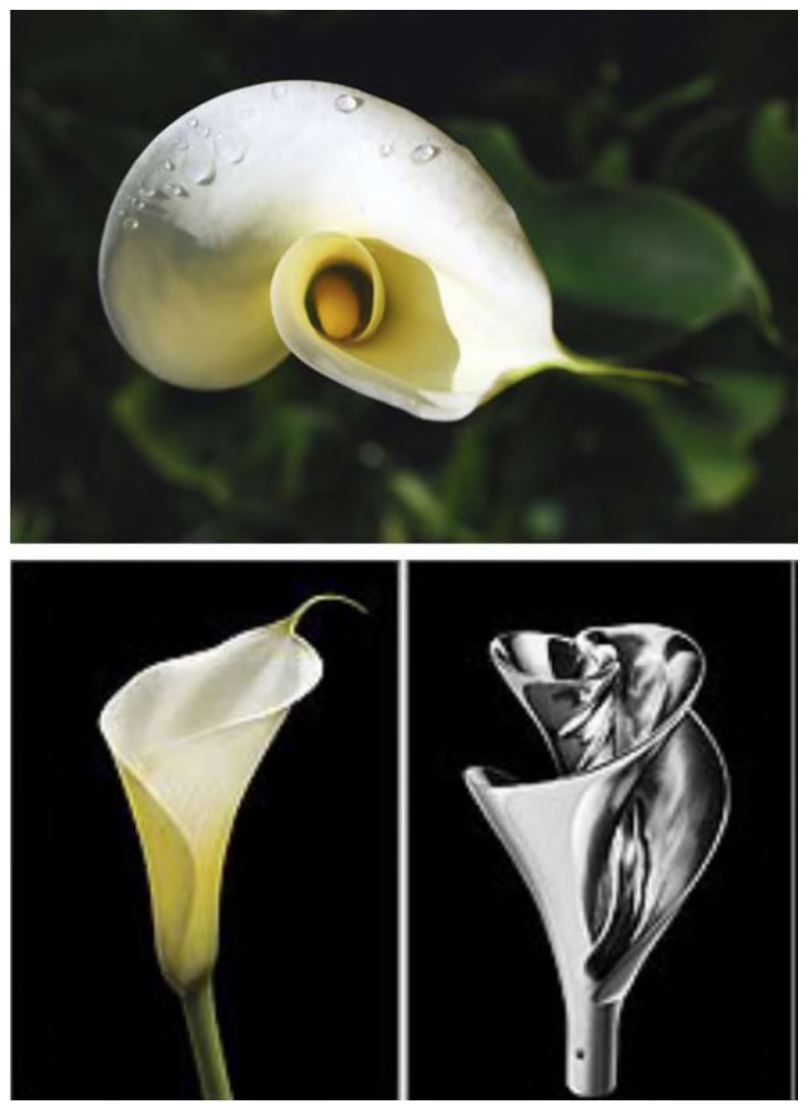

Fig. 7 La cala acumula el agua en su depósito interno

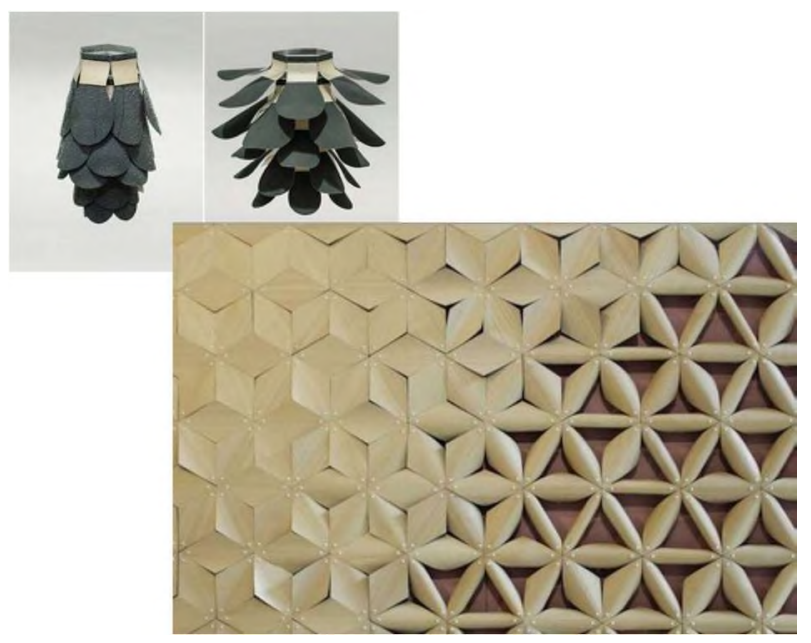

Fig. 8 Chao Chen. Fachada viviente reaccionando a la lluvia

En efecto, su idea nace durante un lluvioso día en Londres, durante el cual el diseñador estaba paseando por Hyde Park.

Esa vez su atención se detuvo en el modo que las piñas en contacto con las gotas de Iluvia alargaban su cáscara externa para proteger sus semillas, una especie de reacción automática que se activa con el contacto con las gotas.

Este mecanismo ha despertado la curiosidad del diseñador haciéndole profundizar la estructura de una piña que el mismo había analizado hasta el mínimo detalle. 
Analizándola se dio cuenta que la piña estaba compuesta por dos cáscaras, uno interno y uno externo: la primera se alarga sobre la segunda cáscara, para impedir que las semillas entren en contacto con el agua de la lluvia. Este mecanismo natural de la piña fue la chispa que ha inspirado a Chao Chen para idear un material en madera laminada capaz de reaccionar al contacto con el agua.

La superficie creada por Chao Chen es biomimética, es decir en función del grado de humedad del aire cambia su forma naturalmente, sin utilizar sistemas electrónicos o estructuras mecánicas, que determinan el movimiento en campo arquitectónico, en cambio, este material permite crear una superficie que cambia de color en contacto con el agua, y es dinámica, es decir que se abre y se cierra para impedir que entre el agua debajo de su superficie. En práctica esta idea se tradujo en una marquesina inteligente que reacciona con la lluvia, compuesta por laminillas que se cierran con el contacto con el agua impidiendo a los usuarios de mojarse en un dia lluvioso.

En los días de sol, las laminillas están abiertas para hacer pasar la luz que es filtrada por estas hojas simuladas, que funcionan como pantalla, así se tendrá la sensación de estar debajo de un árbol. El diseñador ha creado un tercer prototipo, es decir un verdadera superficie arquitectónica que reacciona con la lluvia, pensada en una escala más amplia, por lo tanto un envoltorio de los edificios, como cubierta por ejemplo.

Para hacer superficies tan grandes Chao Chen ha pensado una estructura compuesta por elementos romboidales unidos entre sí a través de pernos, que se asemejan a pétalos de una flor, hechos a propósito con una lámina biomimética.

Estos elementos no deberán estar sujetos a ningún tipo de tratamiento para ser libres de encurvarse y extenderse en función del grado de humedad que hay en el aire, mientras en los días de lluvia la superficie será compacta y cerrada, al contrario en los días de sol tienden a encurvarse dejando pasar las radiaciones solares hacia el interior de los ambientes.

Conceptualmente parecido es el Pabellón experimental Hygroscope, metereo sensible, Achim Menges del Institute for Computational Design, Universidad de Stuttgart en la muestra permanente al Centre Pompidou di Parigi. Se trata de láminas en abeto rojo tratadas en modo de reaccionar cuándo entra en contacto con la humedad y la luz, y de este modo poder abrirse y cerrarse sin necesidad de energía mecánica. (El pabellón está construido completamente en abeto rojo, dividido en 28 cuadrados con un agujero central que permite iluminar toda la estructura, a través de la luz que filtra desde el exterior. La cosa asombrosa es que toda la construcción está regulada sin utilizar controles mecánicos, pero solo a partir de las propiedades naturales de la obra)

Siempre de Achim Menges es el proyecto del Pabellón Elitra, que será colocado en el patio del famoso Victoria and Albert Museum di Londra, cerca de Hyde Park.

La instalación une la biomimesis a las tecnologías emergentes en el campo de la robótica aplicada al diseño, para crear un pabellón que puede modificar en el tiempo la propia estructura. Menges ha trabajado en este proyecto junto al ingeniero estructural Jan Knippers y al ingeniero del clima Thomas Auer. 


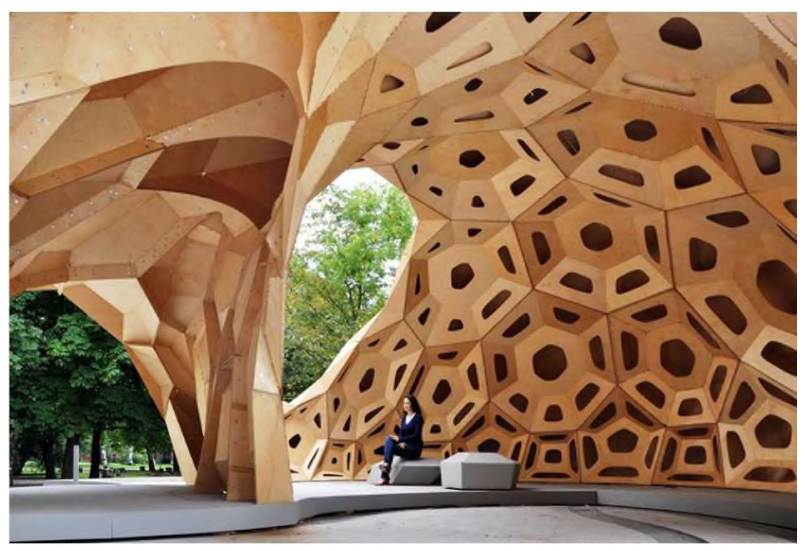

Fig. 9 Pabellón Hygroscope

La idea viene de la estructura típica de las alas del coleóptero, los elitros. El pabellón, rebautizado "Filamento de elitra", consiste en una cobertura ondulada compuesta de fibras de carbón dispuestas según un estrecho trenzado. Para hacerlo se utilizará un brazo robótico que lo "hará girar" directamente en el lugar.

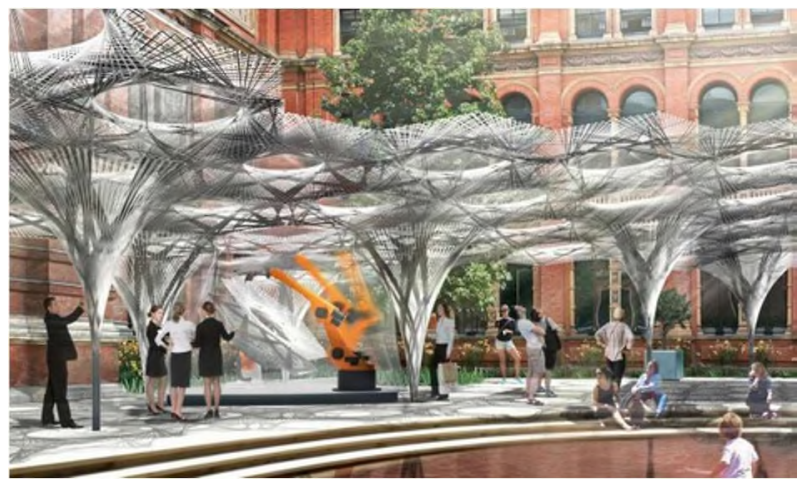

Fig. 10 Pabellón ELITRA, Architect Achim Menges, Victoria and Albert Museum

"Construido enteramente con sistemas fibrosos producidos por un robot - afirma Menges, que también es el fundador del Instituto para el Design Computacional en la Universidad de Stuttgart - el pabellón caracterizará la experiencia del visitante del patio del museo dando un espacio diferenciado y en evolución. Su compleja cobertura filamentosa es al mismo tiempo un envoltorio arquitectónico, una estructura capaz de sostener determinados pesos y un filtro ambiental, que se extenderá y se transformará con el tiempo"

¿Cómo se produce esta transformación? En diferentes puntos del pabellón vienen posicionados sensores. Los datos recogidos por los instrumentos que suministran un cuadro general de la evolución del clima y de las principales características ambientales. Por ejemplo logran percibir la mayor o menor cantidad de luz, las variaciones de temperatura y las diferencias entre una zona y otra del patio. Como respuestas a los cambios entra de nuevo en función el robot, que modificara, expandirá y consecuentemente mejorara la estructura en fibra de carbono del pabellón. Todo aquello que está a la vista del visitante del museo, se podrá ver la transformación en tiempo real en el pabellón.

Aquatic Center de Beijing, realizado en las Olimpíadas del 2008. Del Studio PTW Architect con el Estudio Arup para la estructura tiene un marco en acero cubierto en EFTE (Etilene Tetra Fluoruro Etilene) que imita la estructura de la espuma, de las burbujas de jabón y 
permite un mayor pasaje de la luz y calor que el vidrio tradicional, consintiendo una reducción del $30 \%$ de los costos energéticos.

(La particularidad está en las paredes inspiradas en burbujas. Esta composición garantiza la resistencia antisísmica, además el material plástico permite una mayor iluminación solar con respecto al vidrio y absorbe el calor de los rayos que se transfiere al agua de las piletas.)

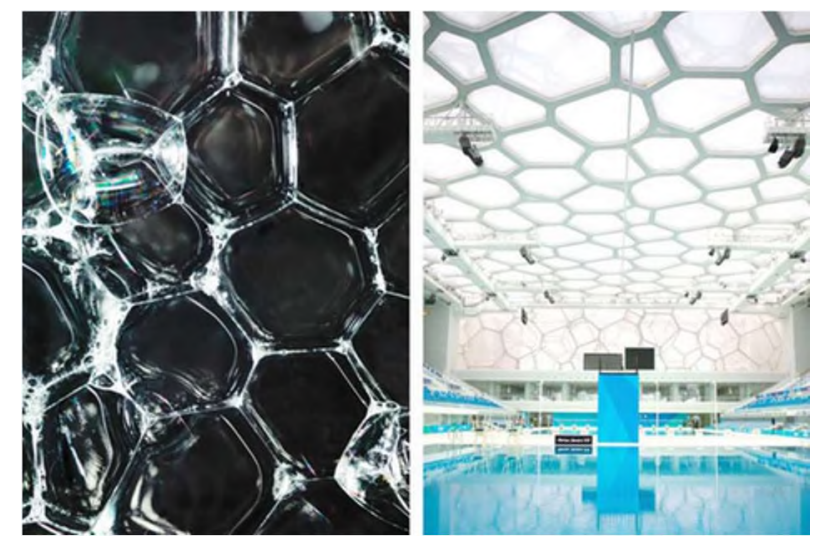

Fig. 11 Beijing national Aquatic Center, 2008, China

El Museo de Historia Natural de Shangai, Studio Perkins Will, está inspirado en el caracol del Nautilus. Tiene 30 metros de altura, y su pared de vidrio está inspirada en la estructura celular de las plantas. La piel inteligente del edificio maximiza el absorbimiento de la energía solar en la $T$ interna y está regulada en un sistema basado en la geotermia, y sobre el confort climático creado por pequeños espejos de agua, por la cobertura verde que además permite recolectar el agua de lluvia para consumo hídrico.

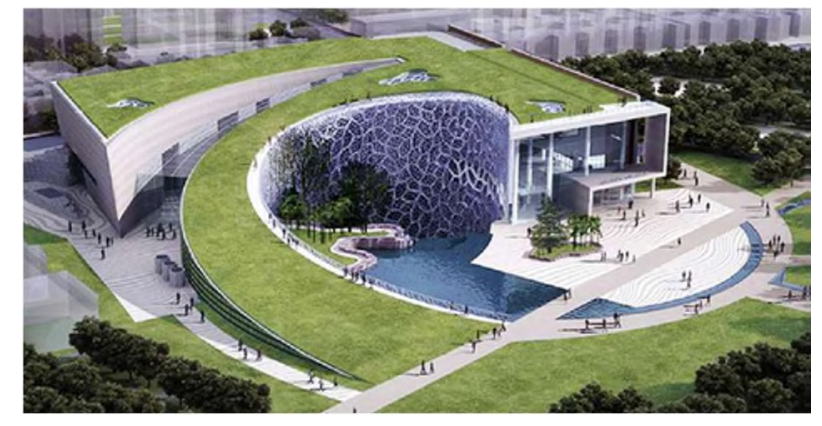

Fig. 12 Museo Historia Natural de Shangai, Studio Perkins Will

BIQ House o Alga House de Hamburgo. Edificio experimental en la International Building Exibition de Hamburgo. La casa-alga proyecto de Splitterwerk Architects de Graz y Estudio Arup es estadio de 5 pisos para 15 unidades habitables con las paredes externas de vidrio que contienen micro algas. ¿Cuáles son los puntos positivos de este proyecto?

El mantenimiento de una temperatura ideal: fresca en verano y sin dispersión del calor en invierno a través del proceso de fotosíntesis de las algas que absorben los rayos solares y el anhídrido carbónico, produciendo energía térmica útil para la calefacción de los ambientes del edificio y agua caliente: en efecto las algas, generan también biomasa (a través de biorreactores) que transformada en metano permite calentar todas las habitaciones y el agua. En un año, este sistema cubre la necesidad de energía de una familia compuesta por 
4 personas. El crecimiento de las algas también produce un sistema de sombra reactivo y natural en los meses del verano.

Aislamiento acústico: estas plantas son un óptimo sistema de insonorización.

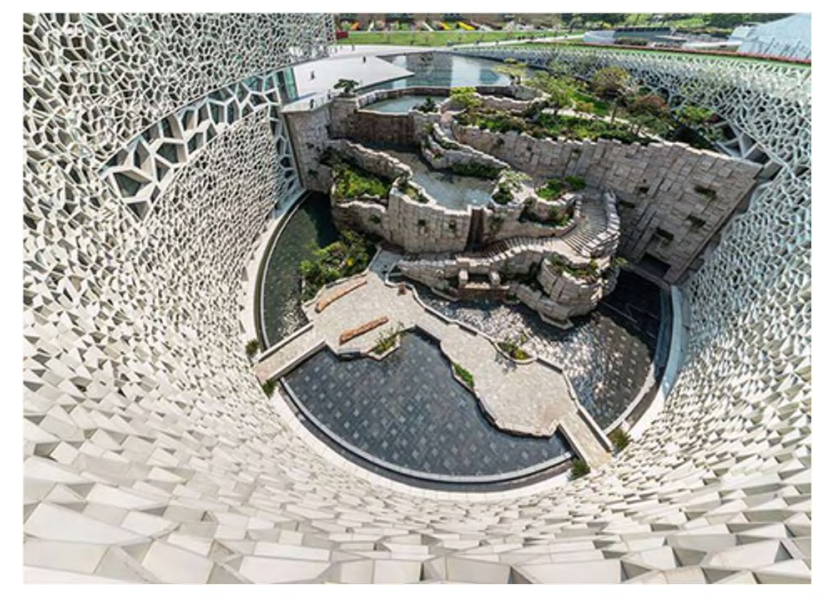

Fig. 13 Museo de Historia Natural de Shangai, Studio Perkins Will

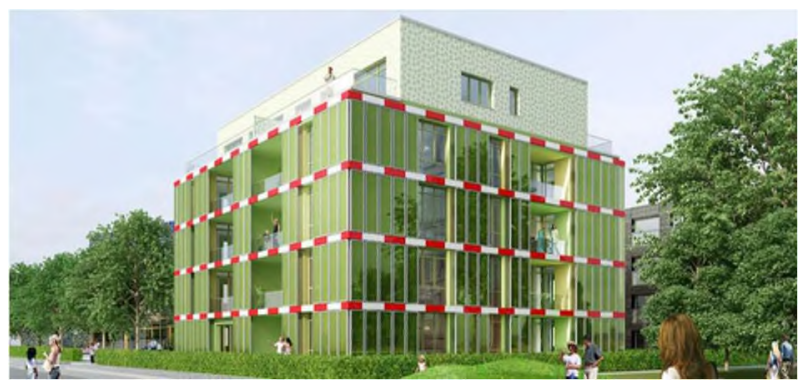

Fig. $14 \mathrm{BIQ}$ House, Hamburgo, fachada bioreactiva con algas

\section{Eastgate Harare Center (1996), Zimbawe, MiKe Pearce con el estudio Arup}

El edificio para la climatización imita el funcionamiento de un hormiguero. Las hormigas necesitan sobrevivir en una temperatura estable de 30 grados y para esto construyen conductos de ventilación que permiten a las cámaras subterráneas mantener tal temperatura gracias a los movimientos conectivos del aire que es aspirado hacia el interior. El proyecto de Mick Pearce está constituido por dos edificios, uno de frente al otro, conectados por una cobertura de vidrio que deja espacio a la circulación del aire. Algunos ventiladores de bajo consumo colocados en el primer piso, aspiran el aire del espacio abierto entre los dos edificios y la bombean en determinados conductos verticales en el interno de dos cuerpos de fábrica, haciéndola atravesar todos los pisos para después desalojarla por las chimeneas que dan al exterior, como sucede en un hormiguero. Este mecanismo tiene una doble función: por un lado garantizar el recambio de aire de los ambientes y por el otro los tiene siempre a temperatura constante.

Con el sistema de enfriamiento natural de un hormiguero, el Eastgate Centre, no teniendo necesidad de un sistema de acondicionamiento artificial, ha hecho ahorrar hasta ahora más de 3,5 millones de dólares y, a lo largo de su vida, continuara a hacer ahorrar más.

Finalmente de la bioimitación toman parte también la arquitectura que se propone no transferir simples mecanismos de funcionamiento de los organismos naturales sino recrear en un ámbito urbano elementos ecosistémicos típicos de ambientes naturales, animales y 
vegetales con el fin de mejorar el confort microclimático urbano y también parcialmente, aumentar indicadores ecosistémicos típicos del ambiente natural como la biodiversidad imitando al bosque.

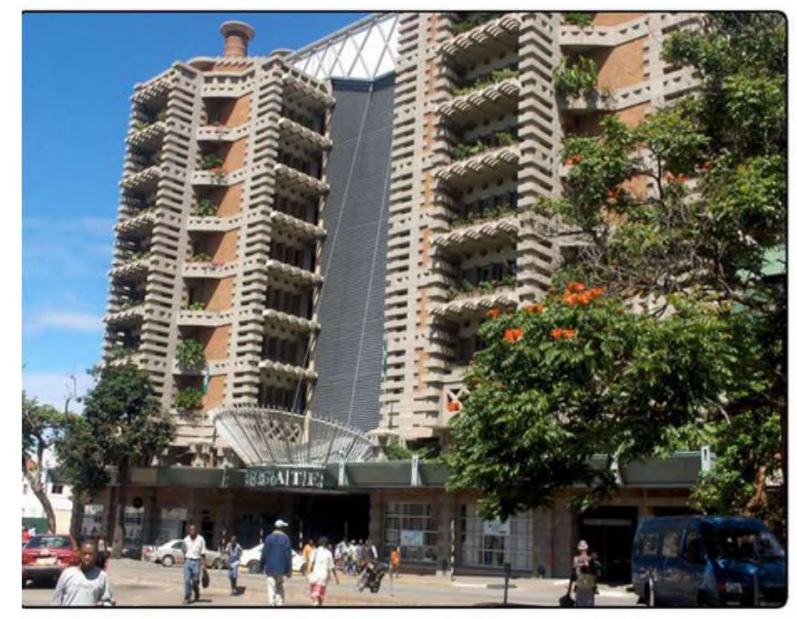

Fig. 15 Eastgate Harare Center (1996), Zimbawe, MiKe Pearce con el estudio Arup

El Bosque Vertical, forma parte de esta categoría, el complejo residencial proyectado por el Estudio Boeri de Milano.

El Bosque vertical es un modelo de edificio residencial sostenible, un proyecto de repoblación forestal de la ciudad que contribuye a la regeneración del ambiente y la bio diversidad urbana sin expandir la ciudad sobre el territorio. Se trata de un modelo de densificación vertical de la naturaleza en el interior de la ciudad, que actúa en relación a la política de reforestación y naturalización de las ciudades. El primer ejemplo de Bosque Vertical, está compuesto por dos torres residenciales de 110 y $76 \mathrm{~m}$ de altura, fue realizado en el centro de Milano, en el límite del barrio Isola, y hospeda 800 árboles (cada uno de estos de 3, 6 o 9 metros), 4.500 arbustos y 15.000 plantas de una vasta gama de arbustos y flores, distribuidos en relación a la posición de la fachada que da al sol. En cada Bosque vertical hay presente una cantidad de árboles que ocuparía una superficie de $20.000 \mathrm{~m} 2$. El sistema vegetal del Bosque Vertical ayuda en la creación de una especie del microclima, produce humedad y oxígeno, absorbe partículas de $\mathrm{CO} 2$ y polvos finos.

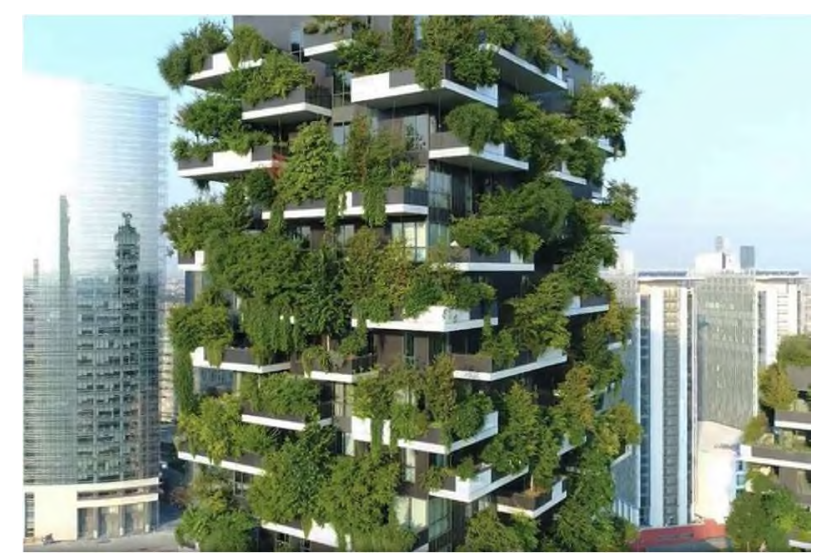

Fig. 16 Bosque vertical Estudio Boeri Milan 
El hábitat biológico del Bosque Vertical aumenta la biodiversidad, ayudando a generar un ecosistema urbano. Los diversos tipos de vegetación crean en efecto un ambiente vertical que puede ser colonizado por pájaros e insectos, transformando el Bosque Vertical en un símbolo de la recolonización espontánea de la ciudad con vegetales y animales. La realización de un cierto número de Bosques Verticales en la ciudad podrá dar vida a una red de pasillos ambientales, que alimentarán el ecosistema principal de parques urbanos, conectando los diferentes espacios de crecimiento de la vegetación espontánea.

El Bosque Vertical es también una medida "anti-sprawl", que tiene como objetivo como controlar y reducir la expansión urbana. Si pensamos a ellos en términos de densificación urbana, cada torre del Bosco Verticale es equivalente a una zona de expansión residencial que puede medir hasta 50.000 metros cuadrados.

El Bosco Verticale es un punto de referencia en la ciudad que permite generar nuevos tipos de paisajes variables, que pueden cambiar su forma en cada estación según la tipología de plantas existentes. La entrada de más Bosques Verticales ofrecerá una visión diversa de la ciudad metropolitana.

En la misma categoría de obras pertenecen las arquitecturas verdes de Patrick Blanc, los muros vegetales $o$ jardines verticales que revisten edificios y estructuras urbanas como el Pont Juvenal en Aix en Provance, que tienen efectos no solo estéticos sobre el pasaje urbano vivo que cambia de color con las estaciones, y también mejoran el microclima urbano, combaten con la isla de calor, con los mecanismos evapotraspirativos de las plantas y aumentan la biodiversidad en el ámbito urbano.

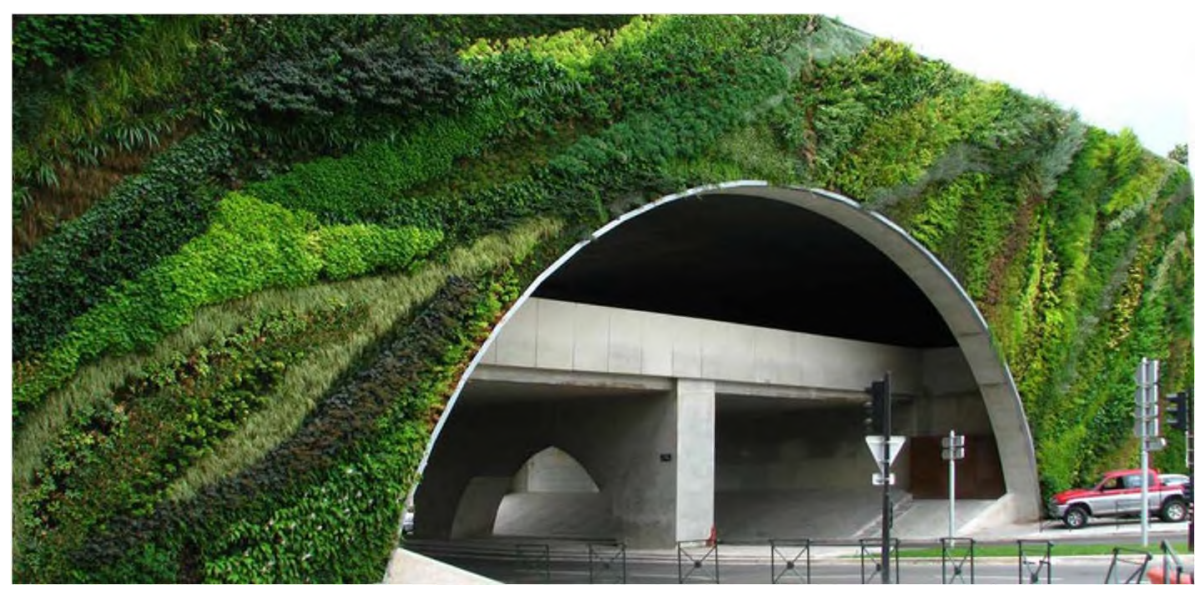

Fig. 17 Green Wall, Pont Juvenal, Aux En Provance, 2008, Patrick Blanc 